\title{
Modèles réduits de grands réseaux avec usines hydroélectriques, et influence du coup de bélier
}

\section{Scale models of major networks, with hydro-electric power stations; study of water-hammer effects}

\author{
PAR I. V. EGUIASAROFF
}

MEMBRE DE L'ACADÉME DES SCIENCES D'ARMÉNIE SOVILTTOVE

\begin{abstract}
Les études de stabilité sur modile réduil peuvent exiger une représentation correcte des installations hydrauliques et des phénomènes de coup de bélier. Le respect des critères de similitude soulève certaines difficultés, par exemple: élasticité de la conduite forcée, influence de l'aération de l'ean sur la viscosité, reproduction des caractéristiques de la tarbine... L'analogie électrique est commode mais pas toujours valable.

L'Institut des Forces Hydrauliques d'Erevan (U.R.S.S.) a réalisé notamment un modèle d'ensemble d'un réseau comprenant trois centrales l'une en analogie hydraulique, les deux autres en analogie électrique.
\end{abstract}

\begin{abstract}
Stability tests on scale models may call for correct reproduction of hydraulic installations and water hammer phenomena. Respecting similitude criteria creates certain difficulties, such as penstock elasticity, effect of water aeration on viscosity and reproduction of turbine characteristics. The electrical analogy is convenient but not always valid.

L'Institut des Forces Hydrauliques d'Erevan, in the U.S.S.R., have produced a model of a network assembly with three power stations, one on hydraulic analogy and the other two on electric analogy.
\end{abstract}

\section{N'TA'TIONS}

$a$ : célérité de l'onde du coup de bélier;

$v_{0}$ : vitesse initiale du courant d'eau;

$\mathrm{H}$ : hauteur de chute;

$\mathrm{H}_{0}$ : chute initiale;

$\mathrm{T}_{s}$ : temps de fermeture (ou d'ouverture) de la turbine;

$\mathrm{L}$ : longueur de la conduite;

$i$ : pente de la ligne de charge hydraulique;

x: échelle du modèle réduit, c'est-à-dire le rapport entre unne grandeur dans l'installation industrielle et la grandeur correspondante dans le modèle, l'indice indiquant l'élément considéré;

D : diamètre de la conduite;

o : épaisseur de la conduite.

Le développement des grands réseaux avec usines hydroélectriques et leur interconnexion croissante ne permettent plus de se contenter d'études à l'échelle industrielle et il a fallu avoir recours aux modèles réduits (microréseaux) en laboratoire, surtout pour les recherches sur les phénomènes transitoires, l'étude des cas d'avaries et de perte de synchronisme, la vérification du comportement des appareils de réglage automatique, ete.

Les premiers laboratoires avec modèles réduits de réseaux entiers ont été créés en U.R.S.S. vers 
la fin de la guerre par Venikov à Moscou [1], Kostenko à Leningrad [2] et l'auteur de cet article à Erevan (R.S.S. d'Arménie) [3]. Actuellement, des recherches de ce genre ont été entreprises dans la plupart des pays $[4,5,6]$. La méthode adoptée en principe pour ces recherches est celle selon laquelle les éléments dynamiques des réseaux sont représentés par des modèles également dynamiques, à savoir des machines tournantes.

Les modèles statiques, les tables électriques à calcul, les machines à calculer recoivent à présent une assez grande application, mais n'ont qu'un rôle secondaire, lorsqu'il s'agit de recherches sur les régimes transitoires pour des réseaux complets.

Pour les réseaux avec usines hydroélectriques, le coup de bélier joue un rôle important dans les questions de stabilité des réseaux, de chan- gements brusques de régime, de pertes de synchronisme et dans ses conséquences sur la qualité du réglage automatique.

C'est ce phénomène du coup de bélier qui a rendu nécessaire de reproduire la partie hydraulique et les effets du coup de bélier dans un même modèle réduit d'un grand réseau.

Le problème que cela pose peut être résolu de deux manières en reproduisant l'effet du coup de bélier soit par analogie électro-dynamique, soit par la méthode physique, c'est-à-dire au moyen d'un modèle réduit hydraulique.

Le problème étudié présente une importance tant pour les usines hydroélectriques avec conduites forcées, que pour les installations sans conduites, mais avec bâches et aspirateurs (pour turbines à réaction), car le coup de bélier est important même dans ce dernier cas.

\section{CRITERES DE SIMILTUDE POUR UN MODËLE REPRODUISANT LE COUP DE BELIER}

Dans des études publiées en 1953 [3], l'auteur a analysé les équations différentielles du coup de bélier et a montré que les paramètres sans dimensions o et $\theta$ d'Allievi [7] constituent des critères de similitude suffisants pour résoudre la question du modèle réduit.

Ces critères sont :

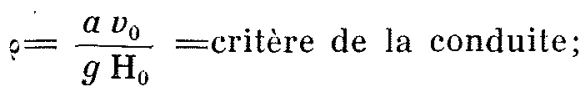

$0=\frac{\mathrm{T}_{s}}{\mathrm{~L} / \mathrm{a}}=\frac{a t}{x}=$ critère de fermeture (ou d'ou-

verture) dit critère homochrone.

Dans le cas plus général du mouvement non permanent dans une conduite, lorsqu'on tient compte $\mathrm{du}$ frottement et du rapport $v / a$, on trouve, en partant des équations différentielles générales, deux critères de similitude de plus [3] :

$$
\begin{aligned}
& \frac{v}{a}=\text { critère de Mach; } \\
& \frac{i_{\text {frot }}}{i}=\text { critère de frottement. }
\end{aligned}
$$

Les conditions pour un modèle hydraulique, lorsque celui-ci ne constitue qu'une partie du modèle complet d'un grand réseau, avec sa partie mécanique (pour le moment d'inertie du groupe générateur) et sa partie électrique (pour l'alternateur, le réseau de distribution et la consom- mation d'énergie électrique) exigent que l'échelle des temps $\alpha_{t}$ soit la même pour tout le réseau et égale à l'unité, $\alpha_{t}=1$.

Il faut que toutes les constantes caractéristiques de temps soient les mêmes sur le modèle et dans l'installation en vraie grandeur.

Pour satisfaire à cette condition, les deux critères de similitude pour le coup de bélier (1) et (2) exigent que :

d'où :

$$
\alpha_{L} \neq \alpha_{H} \neq \alpha_{D} \neq \alpha_{5}
$$

$$
\alpha_{a}=\alpha_{\mathrm{L}}>>1
$$

Ceci signifie que toutes les dimensions géométriques doivent avoir dans le modèle des échelles différentes et que la célérité $a$ de l'onde de coup de bélier doit être, dans le modèle, de dix à vingt fois plus faible que dans l'installation en vraie grandeur.

Ceci ne peut être obtenu que par un changement considérable du module d'élasticité de la conduite forcée du modèle.

Pratiquement, il est possible d'obtenir une telle réduction de la célérité de $a$, en introduisant dans une conduite en acier un tuyau en caoutchouc rempli d'air comprimé. Des recherches très intéressantes sur l'influence d'un tel tuyau rempli d'air ont été faites par Remenieras [8], mais dans un autre but, à savoir, la réduction de la valeur maximum du coup de bélier dans les installations industrielles. 
Une étude théorique de linlluence mutuelle des célérités des ondes dans le tuyau en caoutchouc et dans la conduite même (cette étude vient d'être publiée [18]), a montré que la célérité est pratiquement la même dans le tuyau et dans la conduite et qu'elle est réduite dans le même rapport que celui existant entre les volumes d'air et d'eau.

Dans le laboratoire des réseaux hydroélectriques de l'Institut des Forces Hydranliques de l'Académie des Sciences de la R.S.S. d'Arménie, à Erevan, on a construit en 1955 un tel modele de conduite forcée de $640 \mathrm{~mm}$ de diamètre avec un tuyau en caoutchouc de $160 \mathrm{~mm}$ de diamètre (ce qui correspond à la valeur de 0,05 pour le rapport des volumes d'air et d'eau) ayant une longueur de
$20,5 \mathrm{~m}$, et en $1956 \mathrm{un}$ modèle ayant une longueur de $67,5 \mathrm{~m}$, avec un réservoir de $40 \mathrm{~m}^{2}$ de superficie prévu pour alimenter la conduite et une turhine hydraulique avec modèle de roue conforme à la roue réelle $[3,9]$.

En introduisant un capteur électrique dans l'eau de la conduite et un autre dans l'air du tuyau en caoutchouc, on enregistrait simultanément les pressions en fonction du temps à l'aide d'un oscillographe.

On a constaté que la célérité de l'onde était la même dans la conduite et dans le tuyau. Pour l'installation d'essai décrite plus haut, la célérité était d'environ $60 \mathrm{~m} / \mathrm{s}$, c'est-à-dire quinze fois plus faible que pour la même conduite sans tuyau en caoutchouc rempli d'air.

\section{EFFET DE L'AERATION DU COURANT D'EAU}

Dans certains cas, au laboratoire, les pompes du modèle de conduite forcée alimentaient la turbine avec de l'eau aérée.

Dans certaines de nos expériences, l'aération était telle que, même avec un réservoir à surface libre, la teneur en air dans l'eau était suffisante pour réduire la célérité de l'onde dans la conduite de 900 à environ $600 \mathrm{~m} / \mathrm{s}$, ce qui correspond à un rapport de 0,0005 entre le volume des bulles d'air et celui de l'eau (sans tuyau en caoutchouc).

Mais, en dehors de cet effet sur $a$, il y en avait encore un autre : une grande dissipation d'énergie, une atténuation considérable des oscillations de la surpression due au coup de bélier"; atténuation beaucoup plus grande que dans le cas du tuyau en caoutchouc dans la conduite où le rapport entre les volumes d'air et d'eau était de 0,05.

Les recherches sur la cause d'une telle dissipation d'énergie ont conduit l'auteur à l'attribuer à l'effet de la viscosité dans les conditions du courant d'eau aéré, c'est-à-dire à la seconde viscosité, viscosité voluminaire.

Le compte rendu d'une grande et très intéressante discussion, publié par la Royal Society of London en 1954 [10], a donné la clef de l'énigme. Les articles de Taylor [11] et Davies [12] sur la seconde viscosité pour le cas général de l'eau aérée ont montré, qu'en tenant compte de la compressibilité non seulement des bulles d'air, mais encore de l'eau, on trouve un accroissement de la viscosité qui peut atteindre un maximum de 6.000 à 7.000 fois la viscosité ordinaire.

Ce qui est surprenant, c'est le fait que cet accroissement, qui est nul pour un rapport des volumes d'air et d'eau égal à zéro, croît très vite et atteint le maximum indiqué plus haut pour un rapport de 0,00005 des volumes d'air et d'eau, puis s'abaisse à 2.000-3.000 pour le rapport 0,0005 et tombe à $100-200$ pour le rapport 0,05 .

Cette dernière augmentation de viscosité est négligeable dans le cas du tuyau en caoutchouc plein d'air (rapport des volumes d'air et d'eau : 0,05). Mais elle devient importante pour une aération moindre créée par la pompe sans tuyau en caoutchouc, aération qu'il faut toujours éviter dans les conditions de laboratoire, puisqu'elle ne se produit pas dans de telles proportions dans les conduites forcées des installations industrielles.

Cette influence de la seconde viscosité doit être également notable dans les écoulements à surface libre et à forte pente aux vitesses supracritiques (dépassant de plus de deux fois la vitesse de l'onde), auxquelles on observe une instabilité du courant et la formation d'intumescences produisant des trains d'ondes irrégulières de hauteur croissante, oui se propagent dans la direction du courant et dites «ragues de pente».

C'est peut-être aussi la cause de la persistance du mouvement laminaire que l'on observe aux nombres de Reynolds supérieurs à la valeur critique pour les courants aérés et pour les ondes aérées des écoulements à surface libre.

Jusqu'à présent, cet effet dissipateur dû à la seconde viscosité n'avait été constaté, paraît-il, que dans les phénomènes acoustiques liés aux effets de relaxation. A notre connaissance, on n'a pas encore cherché à le relier aux phénomènes de l'hydraulique, de l'hydrodynamique et à l'atténuation des ondes dans le cas de l'aération.

Des recherches approfondies sur ce sujet seraient souhaitables. 


\section{AUTRES ESSAIS DE REPRESENTATION DU COUP DE BELIER SUR MODËLES RÉDUITS}

Les études bien connues et classiques de Camichel, Eydoux et Gariel [13] ont été le point de départ des recherches expérimentales sur le coup de bélier.

Les principes d'étude du coup de bélier sur modèle proposés par Foch [1.1] et Larras [15] se rapportent au cas purement hydraulique avec $\alpha_{t}>>1$.

A l'Institut pour l'Electromécanique de l'Académie des Sciences de l'U.R.S.S., on a réalisé un modèle de conduite forcée avec introduction des effets du coup de bélier selon une analogie électrique [16]. Chaque fois que l'on peut négliger la compressibilité de l'eau et l'élasticité des parois de la conduite, ce modèle utilise l'analogie des équations différentielles d'un réseau électrique formé de résistances et de capacités, avec les équations du coup de bélier ainsi simplifiées (coup de bélier de masse).

Un tel modèle électrique est beaucoup plus simple à réaliser que le schéma hydraulique décrit plus haut, mais il est limité au cas des oscillations de faible amplitude.

Il faut noter les recherches faites à 'Ioulouse [20] avec représentation, en analogie électrique, des coups de bélier, sans négliger la compressibilité de l'eau et l'élasticité des parois.

Dans les recherches sur les oscillations de puissance des usines hydroélectriques, on n'a pas encore trouvé de moyen pour les représenter par l'analogie électrique et il faut avoir recours au modèle hydraulique.

C'est surtout le cas des turbines à réaction avec aspirateur. D'après des recherches américaines [17], les oscillations de la puissance électrique sont liées aux oscillations de la pression provoquées par des phénomènes hydrauliques. Ce sont des oscillations causées par le vide, la cavitation et les perturbations de l'écoulement dans les aspirateurs des turbines.

\section{MODELLE REDUIT DE TURBINE HYDRAULIQUE}

Dans la méthode de l'analogie électrique, on utilise un moteur électrique à courant continu, avec un dispositif permettant de réaliser la même caractéristique « couple moteur-vitesse de rotation » qu'avec la turbine hydraulique.

Dans la méthode du modèle réduit, la partie hydraulique est représentée par une conduite forcée avec tuyau à air et par une turbine hydraulique avec roues interchangeables selon les divers types de turbines.

Mais s'il s'agit de représenter la turbine seulement par la caractéristique « couple-vitesse » et si le rendement utile de la turbine n'est pas le but des recherches, on peut, avec la même roue à pales orientables (roue Kaplan) et par l'insertion d'un petit anneau approprié, sous les pales, obtenir des caractéristiques « couple-vitesse » d'inclinaison voulue, c'est-à-dire pour tous les types de turbines [19].

Toutefois, pour des recherches approfondies, il faut représenter la roue de la turbine par un modèle de vitesse spécifique donnée avec un modèle parfait de l'aspirateur. Dans ce dernier cas, le modèle de la turbine ne peut pas être universel.

\section{MODELE D'ENSEMBLE D'UN RESEAU HYDROÉNERGÉTIQUE A L'INSTITUT DES FORCES HYDRAULIQUES D'EREVAN}

Ce modèle représente trois usines hydroélectriques, une longue ligne de transport et les installations de divers consommateurs d'énergie $[3,9]$.

L'une des usines hydroélectriques est représentée sur le modèle par une turbine hydraulique à roue universelle, aspirateur et une con- duite forcée avec tuyau à air en caoutchouc, le tout construit conformément aux conditions de similitude exposées plus haut. Pour les deux autres usines, la partie hydraulique est figurée par analogie électrodynamique.

Les alternateurs ont été obtenus par transformation de machines synchrones de type cou- 
rant, mais avec des modifications dans le fer et le cuivre et une compensation de la résistance active par des dispositifs décrits par Venikov [1] et Kostenko [1,2].
Le moment d'inertie peut être modifié dans les trois cas, de manière à faire varier le temps de lancer du groupe entre 6 et 20 secondes.

\section{BIBLIOGRAPHIE}

(1) Venikov (V. A.). - a) Izvestija OTN Akademii Nauk S.S.S.R., 1954, $\mathrm{n}^{\circ} 4 ; b$ ) « La théorie de la simitude et les méthodes physiques pour les modèles réduits », Moscou, 1949.

(2) Kostenko (M. P.). - Izvestija otN Akademii Nauk S.S.S.R., 1953, $\mathrm{n}^{\circ} 12$.

(3) Eguiasaroff (I. V.). - a) Izvestija Akademii Nauk Armjanskoj S.S.R., 1953, $\mathrm{n}^{\circ} 1$; b) Izvestija OTN Akademii Nauk S.S.S.R., 1953, $\mathrm{n}^{\circ} 10 ;$ c) Doklady Akademii Nauk S.S.S.R., 1953, vol. 92, $\mathrm{n}^{\circ \mathrm{s}} 3$ et 5.

(4) Robent (R.). - a) Micromachines et microréseaux. G.I.G.R.E., 1950 , rapport $n^{\circ} 338$; b) Bulletin de la S.F.E., février 1954.

(5) Cahen (F.). - Bulletin de la S.F.E., février 1953.

(6) Elektrotechnik und Maschinenbau, Wien 1956, $\mathrm{n}^{\circ}$ 7-8.

(7) Allievi (L.). - a) Annali della Società degli Ing. ed Arch., 1903, 1913; b) Revue de Mécanique, 1904 $1914 ;$ c) «Théorie du coup de bélier », Paris, Dunod, 1921.

(8) Remexieras (G.). - La. Houille Blanche, 1952.

(9) Bunjatjan (L. B.) et Zoruan. - Izvestija Akademii Nauk Armjanskoj S.S.R., 1956, $\mathrm{n}^{\circ} 4$.
(10) Proceedings Royal Society of London, 1954, vol. 226, p. 1-10.

(11) TAYlon (G.). - a) Proc. Royal Society of London, 1932 , vol. 138 ; b) 1954 , vol. 226 , p. 35 .

(12) Davies (R.). - Proc. Royal Society of London, 1954, vol. 226, p. 39.

(13) Camichel (C.), Eydoux (D.) et Gariel (M.). - Etude théorique et expérimentale des coups de bélier, Toulouse, Privat, 1918.

(14) Focr. - Revue Générale de l'Hydraulique, 1938, $\mathrm{n}^{\circ} 24$.

(15) Lakras (J.). - Revue Générale de l'Hydraulique, $1951, \mathrm{n}^{\circ} 66$.

(16) Voronoy (A. A.), Pervozyanskij (A. A.), Semenov (V.V.). - Izvestija OTN Akademii Nauk S.S.S.R., $1956, \mathrm{n}^{\circ} 1$.

(17) Rheingans (W. J.). - Trans, ASME, 1940, vol. 62.

(18) Egulasaroff, Kartvelishvili, Pervosvanskiu. Izvestija OTN Aliademii Nauk S.S.S.R., 1957, $\mathrm{n}^{\circ} 11$.

(19) Boundatin. - Izvestija OTN Akademii Nauk Armjanskoj S.S.R., 1958, $\mathrm{n}^{\circ} 3$.

(20) Provemal. - Rapport au VII' Congrès de l'A.I.R.H. à Lisbonne, 1957.

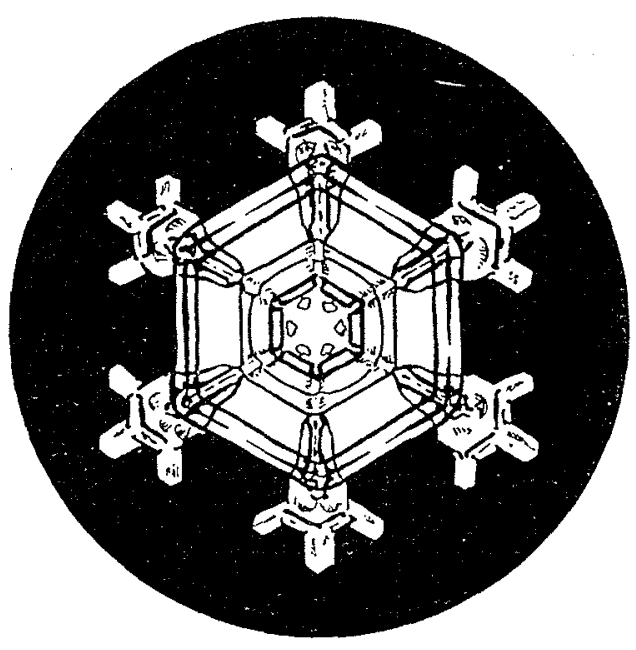

